\title{
Pair production with neutrinos and high-intensity laser fields
}

\author{
Todd M. Tinsley \\ Center for Particle Physics, University of Texas at Austin, Austin, Texas 78712, USA
}

(Dated: November 15, 2018)

\begin{abstract}
We present a detailed calculation of the electron-positron production rate using neutrinos in an intense laser field. The computation is done for the process $\nu \rightarrow \nu e \bar{e}$ via the neutral channel and within the framework of the Standard Model. The production rates are tabulated for a range of incoming neutrino energies in an electromagnetic field similar to what can be attained by current high-intensity lasers.
\end{abstract}

\section{INTRODUCTION}

The introduction of a background electromagnetic field can have very important consequences for neutrino physics. While the Standard Model neutrino does not itself couple to an electromagnetic field, the effects of such a field on neutrinos are made manifest through their interactions with charged particles, real or virtual. Specifically, many investigations have calculated the effects of a constant magnetic field on neutrino propagation and interactions. Recent areas of interest for such calculations include: the neutrino self-energy [1, 2], the $\gamma-\nu-\gamma$ vertex [3], nucleon interactions [4, [5, 6], neutrino-photon scattering [7, 8, 9, 10, 11, 12, 13], and neutrino-electron interactions [14]. A very nice review of these examples and their astrophysical effects has been prepared by Bhattacharya and Pal 15 .

In this article we examine electron-positron pair production through the process $\nu \rightarrow \nu e \bar{e}$. Though normally forbidden, the electromagnetic field alters the final states of the electron-positron pair and frees the interaction to take place. Such a process could have very significant astrophysical and cosmological ramifications. Gvozdev et al. have proposed that this interaction could be important in the analysis of supernovas and neutron star coalescence, and they have offered its role in magnetars as a possible explanation for gamma ray bursts [16]. Such effects are important because of the magnetic field strengths associated with these phenomena. For example, neutron stars and supernovae can have fields in excess of $10^{12} \mathrm{G}$ [17], while a magnetar's field is of order $\lesssim 10^{15} \mathrm{G}[18$.

We, however, turn our focus to examining the production of pairs with neutrinos within more terrestrial fields. We investigate the feasibility of detecting $\nu \rightarrow \nu e \bar{e}$ events using ultrashort pulsed lasers. Though not approaching the astronomical magnetic fields, today's femtosecond terawatt lasers can produce field strengths on the order of $\mathrm{E} \sim 10^{11} \mathrm{~V} / \mathrm{cm}\left(B \sim 10^{9} \mathrm{G}\right)[19]$. We begin by presenting a review of the derivation of the Volkov field operator solution for the electron(positron) in an circularly polarized electromagnetic field (Section III). Next, we apply this Volkov solution to the calculation of the production rate for the process $\nu \rightarrow \nu e \bar{e}$ (Section III). Lastly, we provide a tabulation of production rates for various incoming neutrino energies in the laser field and estimate the likelihood of detecting such a process (Section IV].

\section{DERIVATION OF FIELD}

As was pointed out in Section [ any effect an electromagnetic field has on Standard Model neutrino physics is due to interaction with charged particles in the interaction. For our process we incorporate the laser's intense field into the problem by using the field operator solution of the electron in the presence of an electromagnetic plane wave. We begin by solving the Dirac equation for such a case

$$
\left(\imath \hbar \not \partial-e \not A-m_{\mathrm{e}} c\right) \psi(x)=0 .
$$

We choose the electromagnetic field to be circularly polarized and directed along the $z$-direction such that the 4 -vector potential $A(x)$ is

$$
A(x)=\left(\begin{array}{c}
0 \\
a \cos k \cdot x \\
a \sin k \cdot x \\
0
\end{array}\right)
$$

*Electronic address: ttinsley@physics.utexas.edu 
where $a$ is the magnitude of the vector potential and $\hbar k$ is the 4-momentum directed along the $z$-direction. The choice of circularly polarized light as already been pointed out to be a much simpler approach than assuming linearly or ecliptically polarized light [20, 21].

D. M. Volkov solved the Dirac equation for an electron in the presence of an electromagnetic plane wave 22, 23]. More recent derivations of the solution are outlined in [24, § 40] and [21]. We follow the example of Lifshitz, et al. 24] and find that the Volkov field operator $\psi(x)$ is

$$
\begin{aligned}
\psi(x)= & \int \frac{d^{3} \vec{p}}{(2 \pi)^{3}} \exp \left[\frac{\imath e a}{p \cdot k}\left(p_{1} \sin k \cdot x-p_{2} \cos k \cdot x\right)\right] \sum_{s}\left\{\left(1+\frac{e \not k \not A}{2 p \cdot k}\right) \exp \left[-\frac{\imath}{\hbar}\left(p+\frac{e^{2} a^{2}}{2 p \cdot k} k\right) \cdot x\right] \frac{u^{s}(p)}{\sqrt{2 E_{\vec{p}} / c}} a_{\vec{p}}^{s}\right. \\
& \left.+\left(1-\frac{e \not k A A}{2 p \cdot k}\right) \exp \left[+\frac{\imath}{\hbar}\left(p+\frac{e^{2} a^{2}}{2 p \cdot k} k\right) \cdot x\right] \frac{v^{s}(p)}{\sqrt{2 E_{\vec{p}} / c}} b_{\vec{p}}^{s \dagger}\right\} .
\end{aligned}
$$

In equation (3) we have adopted the notation of [25] where the summation over $s$ is a sum over possible spin states. We integrate over the particle's momentum $p$, and the quantities $p_{1}$ and $p_{2}$ are the components of that momentum along the $x$ and $y$ directions, respectively. We note that the momentum that is to be identified with the Volkov state is not $p$. Instead the Volkov momentum, denoted as $q$, is defined by

$$
q^{\mu}=p^{\mu}+\frac{e^{2} a^{2}}{2 p \cdot k} k^{\mu}
$$

Squaring the state's 4-momentum shows that the mass of the Volkov electron has a dependence on the strength of the electromagnetic field

$$
m_{e}^{* 2}=m_{e}^{2}+e^{2} a^{2} / c^{2}
$$

Note that in the absence of an electromagnetic field $a \rightarrow 0$ the Volkov field operator, equation (3), reduces to the free-field solution

$$
\lim _{a \rightarrow 0} \psi(x)=\int \frac{d^{3} \vec{p}}{(2 \pi)^{3}} \sum_{s}\left\{e^{-\frac{\imath}{\hbar} p \cdot x} \frac{u^{s}(p)}{\sqrt{2 E_{\vec{p}} / c}} a_{\vec{p}}^{s}+e^{+\frac{\imath}{\hbar} p \cdot x} \frac{v^{s}(p)}{\sqrt{2 E_{\vec{p}} / c}} b_{\vec{p}}^{s \dagger}\right\}
$$

and the Volkov mass $m_{e}^{*}$ reduces to the electron mass $m_{e}$.

\section{THE RATE OF PRODUCTION $\Gamma$}

A quantity that we wish calculate for this process is the rate of production $\Gamma$. Physically, the rate of production is the probability per unit time for the neutrino to emit an electron-positron pair in the presence of the laser field. We begin by finding the probability $\mathcal{P}$ for the interaction

$$
\mathcal{P}=\left(\prod_{f} \int \frac{d^{3} \vec{p}_{f}}{(2 \pi)^{3}} \frac{1}{2 E_{f} / c}\right) \int \frac{d^{3} \vec{p}_{\nu}}{(2 \pi)^{3}} \frac{1}{2 E_{\nu} / c} \frac{1}{2} \sum_{s_{\nu}} \sum_{s_{e}, s_{\bar{e}}, s_{\nu^{\prime}}}\left|\left\langle p_{\nu^{\prime}}, s_{\nu^{\prime}} ; p_{e}, s_{e} ; p_{\bar{e}}, s_{\bar{e}}|\hat{S}| p_{\nu}, s_{\nu}\right\rangle\right|^{2}
$$

where we have summed over the spin states of the final neutrino $\nu^{\prime}$, electron $e$, and positron $\bar{e}$, and averaged over the spin states of the incoming neutrino $\nu$. The phase-space integral over the final momentums $p_{e}, p_{\bar{e}}$, and $p_{\nu^{\prime}}$ has been simplified into the form

$$
\prod_{f} \int \frac{d^{3} \vec{p}_{f}}{(2 \pi)^{3}} \frac{1}{2 E_{f} / c}=\int \frac{d^{3} \vec{p}_{\nu^{\prime}}}{(2 \pi)^{3}} \frac{1}{2 E_{\nu^{\prime}} / c} \int \frac{d^{3} \vec{p}_{e}}{(2 \pi)^{3}} \frac{1}{2 E_{e} / c} \int \frac{d^{3} \vec{p}_{\bar{e}}}{(2 \pi)^{3}} \frac{1}{2 E_{\bar{e}} / c}
$$

The scattering operator $\hat{S}$ for our process is

$$
\begin{aligned}
\hat{S}= & -\frac{\imath}{\hbar^{8}} \frac{4 \pi \alpha}{2^{2} \cos ^{2} \theta_{\mathrm{W}} \sin ^{2} \theta_{\mathrm{W}}} \int d^{4} x \bar{\psi}_{e}(x) \gamma^{\mu}\left(-\frac{1}{2}+2 \sin ^{2} \theta_{\mathrm{W}}+\frac{1}{2} \gamma^{5}\right) \psi_{e}(x) Z_{\mu}(x) \\
& \times \int d^{4} y \bar{\psi}_{\nu}(y) \gamma^{\sigma}\left(\frac{1}{2}-\frac{1}{2} \gamma^{5}\right) \psi_{\nu}(y) Z_{\sigma}(y)
\end{aligned}
$$


where $\psi_{e}(x)$ is the Volkov field operator for the electron (equation (3) ), $\psi_{\nu}(y)$ is the free-field operator for the neutrino, and $Z_{\mu}(x)$ is the field operator of the $Z$ boson. $\alpha$ is the fine-structure constant, and $\theta_{\mathrm{W}}$ is the weak-mixing angle.

In the free-field case the integration over the space-time variables results in a 4-dimensional $\delta$-function that conserves energy and momentum. Though this is true of our integral over the space-time coordinate $y$ in equation (9), it is not true for the integral over $x$. The Volkov fields $\psi_{e}(x)$ in the $\int d^{4} x$ integral have a more complicated space-time dependence.

We begin by expanding the field operators and integrating out the $y$ dependence. The scattering matrix can be written as

$$
\begin{aligned}
S= & \left\langle p_{\nu^{\prime}}, s_{\nu^{\prime}} ; p_{e}, s_{e} ; p_{\bar{e}}, s_{\bar{e}}|\hat{S}| p_{\nu}, s_{\nu}\right\rangle \\
S= & -\frac{\imath}{\hbar^{4}} \frac{4 \pi \alpha}{2^{2} \cos ^{2} \theta_{\mathrm{W}} \sin ^{2} \theta_{\mathrm{W}}}\left\{\bar{u}_{s_{\nu^{\prime}}}\left(p_{\nu^{\prime}}\right) \gamma^{\sigma}\left(\frac{1}{2}-\frac{1}{2} \gamma^{5}\right) u_{s_{\nu}}\left(p_{\nu}\right)\right\} \frac{g_{\mu \sigma}-\left(p_{\nu}-p_{\nu^{\prime}}\right)_{\mu}\left(p_{\nu}-p_{\nu^{\prime}}\right)_{\sigma} /\left(m_{Z} c\right)^{2}}{\left(p_{\nu}-p_{\nu^{\prime}}\right)^{2}-\left(m_{Z} c\right)^{2}+\imath \Gamma_{Z} m_{Z} c^{2}} \\
& \times \int d^{4} x\left\{\bar{u}_{s_{e}}\left(p_{e}\right)\left(1+\frac{e \not \not k}{2 p_{e} \cdot k}\right) \gamma^{\mu}\left(-\frac{1}{2}+2 \sin ^{2} \theta_{\mathrm{W}}+\frac{1}{2} \gamma^{5}\right)\left(1-\frac{e \not k A}{2 p_{\bar{e}} \cdot k}\right) \bar{u}_{s_{\bar{e}}}\left(p_{\bar{e}}\right)\right\} \\
& \times \exp \left[\frac{\imath}{\hbar}\left(q_{e}+q_{\bar{e}}+p_{\nu^{\prime}}-p_{\nu}\right) \cdot x\right] \exp \left[-\left(\frac{e a}{p_{e} \cdot k} p_{e_{1}}-\frac{e a}{p_{\bar{e}} \cdot k} p_{\bar{e} 1}\right) \sin (k \cdot x)+\left(\frac{e a}{p_{e} \cdot k} p_{e_{2}}-\frac{e a}{p_{\bar{e}} \cdot k} p_{\bar{e} 2}\right) \cos (k \cdot x)\right] .
\end{aligned}
$$
26.

To fashion equation (10) into an analytically integrable form, we employ Hansen's definition of the Bessel function

$$
e^{(z / 2)(t-1 / t)}=\sum_{n=-\infty}^{\infty} J_{n}(z) t^{n}
$$

We can recast the left-hand side of equation (11) in a fashion more applicable to the exponentials found in equation (10)

$$
e^{-\imath \zeta \sin (k \cdot x-\delta)}=\sum_{n=-\infty}^{\infty} e^{-\imath n k \cdot x} e^{\imath n \delta} J_{n}(\zeta)
$$

where

$$
\begin{aligned}
\zeta \cos \delta & =\left(\frac{e a}{p_{e} \cdot k} p_{e_{1}}-\frac{e a}{p_{\bar{e}} \cdot k} p_{\bar{e} 1}\right), \text { and } \\
\zeta \sin \delta & =\left(\frac{e a}{p_{e} \cdot k} p_{e_{2}}-\frac{e a}{p_{\bar{e}} \cdot k} p_{\bar{e}_{2}}\right) .
\end{aligned}
$$

Since the vector potential $A$ in the $S$-matrix has components that go as $\sin (k \cdot x)$ and $\cos (k \cdot x)$, we will also need to perform a similar expansion for terms like

$$
\left\{\begin{array}{c}
\sin (k \cdot x) \\
\cos (k \cdot x) \\
\sin ^{2}(k \cdot x) \\
\sin (k \cdot x) \cos (k \cdot x) \\
\cos ^{2}(k \cdot x)
\end{array}\right\} \times e^{-\imath \zeta \sin (k \cdot x-\delta)} .
$$

All of these terms, when expanded, result in forms similar to equation (12). The space-time dependence is factored out into an exponential that goes as $e^{-\imath n k \cdot x}$. As an example,

$$
\sin (k \cdot x) e^{-\imath \zeta \sin (k \cdot x-\delta)}=\sum_{n=-\infty}^{\infty} \frac{1}{2 \imath} e^{-\imath n k \cdot x}\left(e^{\imath(n+1) \delta} J_{n+1}(\zeta)-e^{\imath(n-1) \delta} J_{n-1}(\zeta)\right)
$$

After these expansions are made, the space-time integral in the $S$-matrix reduces to the normal 4 -dimensional $\delta$-function

$$
\begin{aligned}
& S \propto \int d^{4} x \exp \left[\frac{\imath}{\hbar}\left(q_{e}+q_{\bar{e}}+p_{\nu^{\prime}}-p_{\nu}-n k\right) \cdot x\right] \\
& S \propto \delta^{4}\left(q_{e}+q_{\bar{e}}+p_{\nu^{\prime}}-p_{\nu}-n k\right) .
\end{aligned}
$$


Physically, equation (15) suggests that it is the Volkov states that are to be considered when conserving momentum and energy. Equation (15) also suggests that we interpret the $n k$ term as a number of photons that are pulled from the laser field in order to drive this reaction. These photons are what allow this process to overcome the energy and momentum constraints that originally prohibited this reaction in the free-field case. In fact, the $\delta$-function can be used to apply a constraint on the possible values for $n$

$$
\begin{aligned}
p_{\nu}{ }^{\mu}+n k^{\mu} & =q_{e}{ }^{\mu}+q_{\bar{e}}{ }^{\mu}+p_{\nu^{\prime}}{ }^{\mu} \\
\left(p_{\nu}{ }^{\mu}+n k^{\mu}\right)^{2} & =\left(q_{e}{ }^{\mu}+q_{\bar{e}}{ }^{\mu}+p_{\nu^{\prime}}{ }^{\mu}\right)^{2} \\
2 n p_{\nu} \cdot k & \geq 4\left(m_{e}^{*} c\right)^{2} .
\end{aligned}
$$

In the proceeding calculation we will take the neutrino and laser beams to be oppositely directed. Therefore, in the massless limit of the neutrino the constraint equation (16) becomes

$$
\begin{aligned}
4 n E_{\nu} E_{\gamma} & \geq 4\left(m_{e}^{*} c\right)^{2} \\
n & \geq \frac{\left(m_{e}^{*} c\right)^{2}}{E_{\nu} E_{\gamma}}
\end{aligned}
$$

where $E_{\gamma}$ is the energy carried per photon. We note that the reaction cannot emit photons $(n \nless 0)$, nor can it proceed without absorbing some number of photons $(n \neq 0)$.

The $S$-matrix can now be rewritten as

$$
S=\imath(2 \pi)^{4} \sum_{n=-\infty}^{\infty} \delta^{4}\left(q_{e}+q_{\bar{e}}+p_{\nu^{\prime}}-p_{\nu}-n k\right) \mathcal{M}_{n}
$$

where we have absorbed the couplings, spinors, Bessel functions, etc., into the scattering matrix $\mathcal{M}_{n}$. For a definition of $\mathcal{M}_{n}$ refer to the Appendix.

Of importance, however, is the square of the $S$-matrix. We begin by substituting the $S$-matrix (equation (18) ) into the probability (equation (7))

$$
\begin{aligned}
\mathcal{P}= & \left(\prod_{f} \int \frac{d^{3} \vec{p}_{f}}{(2 \pi)^{3}} \frac{1}{2 E_{f} / c}\right) \int \frac{d^{3} \vec{p}_{\nu}}{(2 \pi)^{3}} \frac{1}{2 E_{\nu} / c} \\
& \times \frac{1}{2} \sum_{s_{\nu}} \sum_{s_{e}, s_{\bar{e}}, s_{\nu^{\prime}}} \sum_{n, m=-\infty}^{\infty}(2 \pi)^{8} \delta^{4}\left(q_{e}+q_{\bar{e}}+p_{\nu^{\prime}}-p_{\nu}-n k\right) \delta^{4}\left(q_{e}+q_{\bar{e}}+p_{\nu^{\prime}}-p_{\nu}-m k\right) \mathcal{M}_{m}^{*} \mathcal{M}_{n} .
\end{aligned}
$$

By inspection, one can see that the two 4-dimensional $\delta$-functions imply that for there to be any contribution to the summation, either there is no incoming photon energy $\left(E_{\gamma}=0\right)$ or, more appropriately, that $m=n$. Therefore, we can replace $\mathcal{M}_{m}^{*} \mathcal{M}_{n}$ with the square of the norm of the scattering amplitude $\left|\mathcal{M}_{n}\right|^{2}$ and eliminate the sum over $m$

$$
\mathcal{P}=\left(\prod_{f} \int \frac{d^{3} \vec{p}_{f}}{(2 \pi)^{3}} \frac{1}{2 E_{f} / c}\right) \int \frac{d^{3} \vec{p}_{\nu}}{(2 \pi)^{3}} \frac{1}{2 E_{\nu} / c} \sum_{n=-\infty}^{\infty}(2 \pi)^{8}\left(\delta^{4}\left(q_{e}+q_{\bar{e}}+p_{\nu^{\prime}}-p_{\nu}-n k\right)\right)^{2} \overline{\left|\mathcal{M}_{n}\right|^{2}}
$$

To simplify the result, we have defined the square of the scattering matrix after summing and averaging over spins to be

$$
\overline{\left|\mathcal{M}_{n}\right|^{2}}=\frac{1}{2} \sum_{s_{\nu}} \sum_{s_{e}, s_{\bar{e}}, s_{\nu^{\prime}}}\left|\mathcal{M}_{n}\right|^{2}
$$

Next, we can use three of the $\delta$-functions to eliminate the integral over the momentum of the incoming neutrino

$$
\begin{aligned}
\mathcal{P}= & \left(\prod_{f} \int \frac{d^{3} \vec{p}_{f}}{(2 \pi)^{3}} \frac{1}{2 E_{f} / c}\right) \frac{1}{2 E_{\nu} / c} \\
& \times \sum_{n=-\infty}^{\infty}(2 \pi)^{5} \delta^{4}\left(q_{e}+q_{\bar{e}}+p_{\nu^{\prime}}-p_{\nu}-n k\right) \delta\left(\left(E_{e}+E_{\bar{e}}+E_{\nu^{\prime}}-E_{\nu}-n E\right) / c\right) \overline{\left|\mathcal{M}_{n}\right|^{2}}
\end{aligned}
$$


or

$$
\begin{aligned}
\mathcal{P}= & \left(\prod_{f} \int \frac{d^{3} \vec{p}_{f}}{(2 \pi)^{3}} \frac{1}{2 E_{f} / c}\right) \frac{1}{2 E_{\nu} / c} \\
& \times \sum_{n=-\infty}^{\infty}(2 \pi)^{5} \delta^{3}\left(\vec{q}_{e}+\vec{q}_{\bar{e}}+\vec{p}_{\nu^{\prime}}-\vec{p}_{\nu}-n \vec{k}\right)\left(\delta\left(\left(E_{e}+E_{\bar{e}}+E_{\nu^{\prime}}-E_{\nu}-n E\right) / c\right)\right)^{2} \frac{\left|\mathcal{M}_{n}\right|^{2}}{}
\end{aligned}
$$

This still leaves us with two identical energy-conserving $\delta$-functions. To simplify we follow the solution pointed out by [21] and outlined in 27]. We write one of these $\delta$-functions as an integral over time

$$
\begin{aligned}
& \mathcal{P} \propto \sum_{n=-\infty}^{\infty}\left(\delta\left(\left(E_{e}+E_{\bar{e}}+E_{\nu^{\prime}}-E_{\nu}-n E\right) / c\right)\right)^{2} \\
& \mathcal{P} \propto \sum_{n=-\infty}^{\infty} \delta\left(\left(E_{e}+E_{\bar{e}}+E_{\nu^{\prime}}-E_{\nu}-n E\right) / c\right) \frac{c}{2 \pi \hbar} \lim _{T \rightarrow \infty} \int_{-T / 2}^{T / 2} d t e^{\frac{\imath}{\hbar}\left(E_{e}+E_{\bar{e}}+E_{\nu^{\prime}}-E_{\nu}-n E\right) t}
\end{aligned}
$$

and use the other $\delta$-function to reduce the integrand to unity

$$
\begin{aligned}
& \mathcal{P} \propto \sum_{n=-\infty}^{\infty} \delta\left(\left(E_{e}+E_{\bar{e}}+E_{\nu^{\prime}}-E_{\nu}-n E\right) / c\right) \frac{c}{2 \pi \hbar} \lim _{T \rightarrow \infty} \int_{-T / 2}^{T / 2} d t \\
& \mathcal{P} \propto \sum_{n=-\infty}^{\infty} \delta\left(\left(E_{e}+E_{\bar{e}}+E_{\nu^{\prime}}-E_{\nu}-n E\right) / c\right) \frac{c}{2 \pi \hbar} \lim _{T \rightarrow \infty} T .
\end{aligned}
$$

By making the substitution of equation (23) into the probability in equation (22)

$$
\mathcal{P}=\lim _{T \rightarrow \infty}\left(\prod_{f} \int \frac{d^{3} \vec{p}_{f}}{(2 \pi)^{3}} \frac{1}{2 E_{f} / c}\right) \frac{1}{2 E_{\nu} / c} \frac{c}{2 \pi \hbar} T \sum_{n=-\infty}^{\infty}(2 \pi)^{5} \delta^{4}\left(q_{e}+q_{\bar{e}}+p_{\nu^{\prime}}-p_{\nu}-n k\right) \overline{\left|\mathcal{M}_{n}\right|^{2}}
$$

we can solve for the quantity of interest in this problem, the rate of production $\Gamma$

$$
\begin{aligned}
\Gamma & =\frac{d \mathcal{P}}{d t} \rightarrow \frac{\mathcal{P}}{T} \\
\Gamma & =\frac{c}{\hbar}\left(\prod_{f} \int \frac{d^{3} \vec{p}_{f}}{(2 \pi)^{3}} \frac{1}{2 E_{f} / c}\right) \frac{1}{2 E_{\nu} / c} \sum_{n=-\infty}^{\infty}(2 \pi)^{4} \delta^{4}\left(q_{e}+q_{\bar{e}}+p_{\nu^{\prime}}-p_{\nu}-n k\right) \overline{\left|\mathcal{M}_{n}\right|^{2}}
\end{aligned}
$$

It may be most appropriate to think of the total production rate $\Gamma$ as the sum of the production rates for all of the individual processes involving $n$ photons

$$
\Gamma=\sum_{n=-\infty}^{\infty} \Gamma_{n}
$$

where

$$
\Gamma_{n}=\frac{c}{\hbar}\left(\prod_{f} \int \frac{d^{3} \vec{p}_{f}}{(2 \pi)^{3}} \frac{1}{2 E_{f} / c}\right) \frac{1}{2 E_{\nu} / c}(2 \pi)^{4} \delta^{4}\left(q_{e}+q_{\bar{e}}+p_{\nu^{\prime}}-p_{\nu}-n k\right) \overline{\left|\mathcal{M}_{n}\right|^{2}}
$$

\section{RESULTS}

Our calculation proceeds by computing the individual production rates $\Gamma_{n}$ (equation (26) ) and then summing to find the total rate $\Gamma$ (equation (25)). Though the constraint in equation (17) imposes a lower bound on the summation over $n$ for the total rate, there is no such upper bound. This means that the calculation should include computations of the individual production rates for $n$ out to infinity. Fortunately, the individual production rates $\Gamma_{n}$ fall off exponentially 


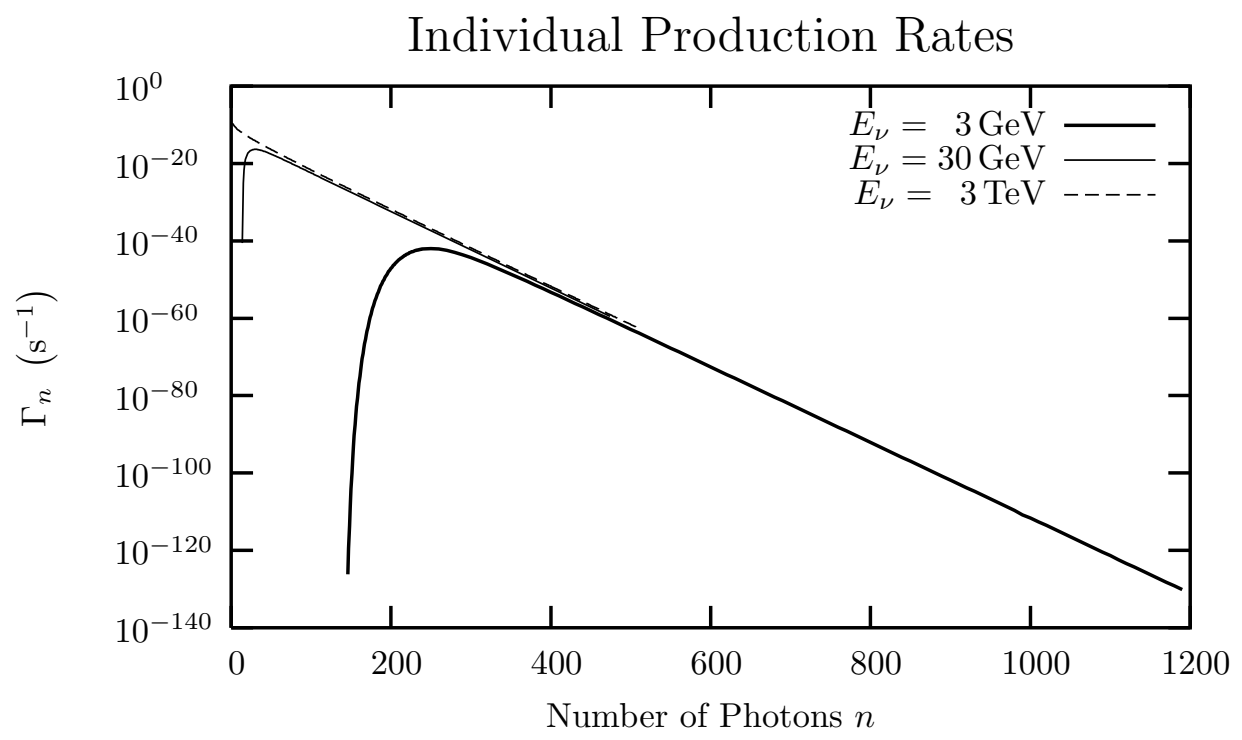

FIG. 1: The profile of the individual production rates $\Gamma_{n}$ as a function of $n$ for incoming neutrino energies of $3 \mathrm{GeV}, 30 \mathrm{GeV}$, and $3 \mathrm{TeV}$.

for large $n$. How fast (or slowly) these rates fall off depends on what choices are made for the incoming photon energy $E_{\gamma}$ and the magnitude of the vector potential $a$. For the cases in which the individual rates fall off slowly, we calculate the rates to sufficiently large $n$ such that we can characterize the nature of the exponential decay. Using this characterization to fit the rates at large $n$ allows us to approximate the summation.

We choose initial conditions that closely approximate today's high-intensity laser system. These conditions are based on the Terawatt High-intensity Optical Research (THOR) laser at the High Intensity Laser Science Group at the University of Texas at Austin. The THOR laser is a $20 \mathrm{TW}$ laser beam centered around a wavelength of $800 \mathrm{~nm}$ with an electric field strength $|\vec{E}|$ on the order of $10^{10} \sim 10^{11} \mathrm{~V} / \mathrm{cm}[19]$. For our calculations we choose an electric field strength of $|\vec{E}|=5 \cdot 10^{10} \mathrm{~V} / \mathrm{cm}$. The choice of wavelength and electric field strength uniquely specify the electromagnetic field parameters in our problem. Using the customary relationships, the photon energy is

$$
E_{\gamma}=h c / \lambda,
$$

and the magnitude of the vector potential is

$$
a=|\vec{E}| \hbar / E_{\gamma} .
$$

The profile of the individual production rates $\Gamma_{n}$ as a function of the number of photons $n$ is given in Fig. 目 for incoming neutrino energies of $3 \mathrm{GeV}, 30 \mathrm{GeV}$, and $3 \mathrm{TeV}$. Notice that for a given choice of photon energy and vector potential magnitude, the profiles shown in Fig. 1 all follow the same exponential decay.

The individual rates that make up these profiles are summed in order to find the total production rate (equation (25)). We present the results of this summation in Table 1 for a range of neutrino energies from $1 \mathrm{GeV}$ to $300 \mathrm{TeV}$. The total rate of production is also presented for a laser field that is able to generate the same field strengths but with a much smaller wavelength of light $\lambda=100 \mathrm{~nm}$. We note that the difference in wavelengths has the most significant effect at low incoming neutrino energies.

Rather than considering the total rate of production for this process, it may have more physical significance if we consider the production length $\Lambda$. The production length is the distance that a neutrino must travel in the laser field such that its likelihood of producing an electron-positron pair is $1-1 / e \simeq 63 \%$. That is, the probability for production is

$$
\mathcal{P}=1-e^{-\ell / \Lambda}
$$

where $\ell$ is the distance traveled in the field, and the production length is simply $\Lambda=c / \Gamma$. The production lengths for the rates tabulated in Table \are shown in Fig. 2

From inspection of Fig. 22 one can see how feeble this interaction is. For the production length to drop below the Hubble length, an estimate for the size of the universe, the incoming neutrino energy must exceed $10 \mathrm{GeV}$. And at energies near $1 \mathrm{PeV}$ the production length is still on the order of a light year. 


\begin{tabular}{|r||l|l|}
\hline \multicolumn{1}{|c||}{$E_{\nu}$} & \multicolumn{2}{c|}{$\Gamma\left(\mathrm{s}^{-1}\right)$} \\
\cline { 2 - 3 } & $\lambda=800 \mathrm{~nm}$ & $\lambda=100 \mathrm{~nm}$ \\
\hline $1 \mathrm{GeV}$ & $9.1 \cdot 10^{-93}$ & $6.7 \cdot 10^{-58}$ \\
$3 \mathrm{GeV}$ & $3.3 \cdot 10^{-41}$ & $8.1 \cdot 10^{-29}$ \\
$10 \mathrm{GeV}$ & $3.8 \cdot 10^{-22}$ & $1.1 \cdot 10^{-17}$ \\
$30 \mathrm{GeV}$ & $6.7 \cdot 10^{-16}$ & $8.3 \cdot 10^{-14}$ \\
$100 \mathrm{GeV}$ & $5.5 \cdot 10^{-13}$ & $8.1 \cdot 10^{-12}$ \\
$300 \mathrm{GeV}$ & $1.3 \cdot 10^{-11}$ & $5.7 \cdot 10^{-11}$ \\
$1 \mathrm{TeV}$ & $1.2 \cdot 10^{-10}$ & $3.0 \cdot 10^{-10}$ \\
$3 \mathrm{TeV}$ & $6.0 \cdot 10^{-10}$ & $1.2 \cdot 10^{-9}$ \\
$10 \mathrm{TeV}$ & $2.9 \cdot 10^{-9}$ & $5.1 \cdot 10^{-9}$ \\
$30 \mathrm{TeV}$ & $1.1 \cdot 10^{-8}$ & $1.8 \cdot 10^{-8}$ \\
$100 \mathrm{TeV}$ & $4.8 \cdot 10^{-8}$ & $7.1 \cdot 10^{-8}$ \\
$300 \mathrm{TeV}$ & $1.7 \cdot 10^{-7}$ & $2.4 \cdot 10^{-7}$ \\
\hline
\end{tabular}

TABLE I: The total production rate $\Gamma$ for a given incoming neutrino energy $E_{\nu}$ in the presence of an laser field of electric field strength $|\vec{E}|=5 \cdot 10^{10} \mathrm{~V} / \mathrm{cm}$ and wavelength $\lambda$.

\section{Production Length}

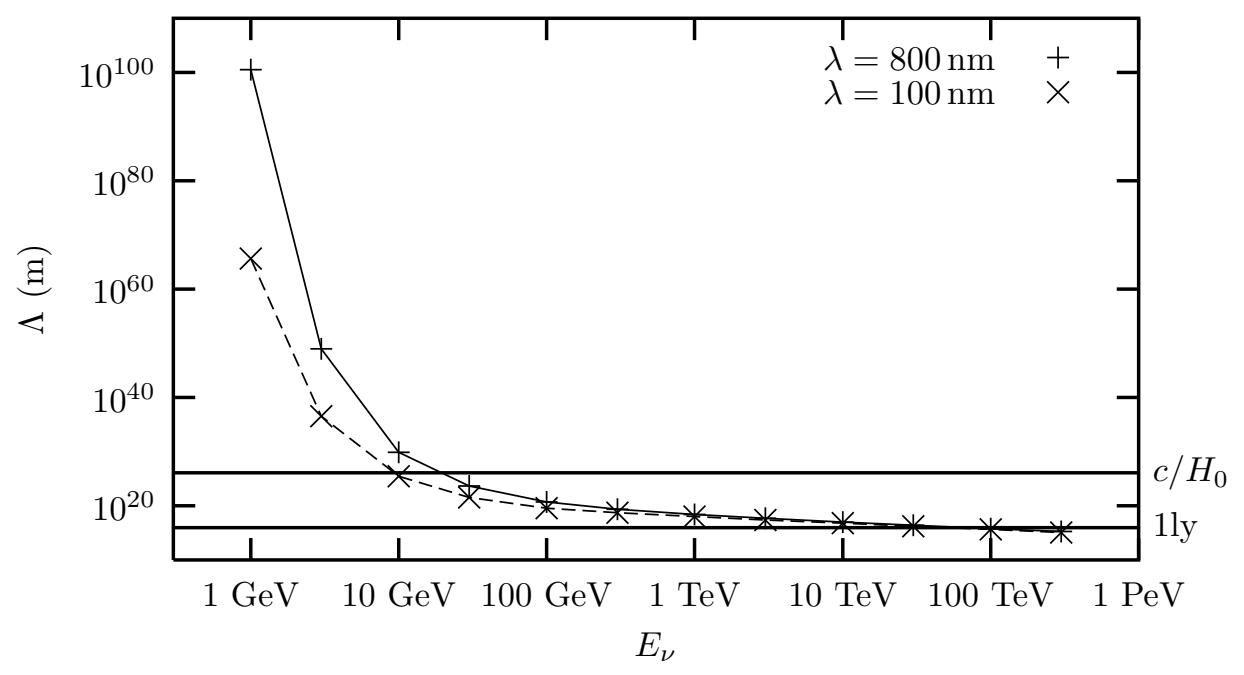

FIG. 2: The production lengths $\Lambda$ as a function of incoming neutrino energy $E_{\nu}$ for a laser field of wavelengths $100 \mathrm{~nm}$ and $800 \mathrm{~nm}$ at an electric field strength of $|\vec{E}|=5 \cdot 10^{10} \mathrm{~V} / \mathrm{cm}$. The dark lines correspond to the Hubble length $c / H_{0} \sim 1.2 \cdot 10^{26} \mathrm{~m}$ and one light year $1 \mathrm{ly}=9.46 \cdot 10^{15} \mathrm{~m}$.

To further stress the small likelihood of pair production under these conditions, we can consider this interaction at a neutrino source such as the Neutrinos at the Main Injector (NuMI) facility at Fermilab. If we assume the most ideal set of specifications for the neutrino beam [28] and simplify the beam to be uniform, we estimate the neutrino flux to be on the order of $10^{19} / \mathrm{m}^{2} / \mathrm{s}$ per pulse with a pulse every $2 \mathrm{~s}$. The energy profile of the beam is nontrivial. There is a low-energy component peaked at $3 \mathrm{GeV}$, a medium-energy component peaked at $7 \mathrm{GeV}$, and a high-energy component peaked at $15 \mathrm{GeV}$, but the beam will contain neutrinos with energy up to $120 \mathrm{GeV}$ [29]. However, for estimation purposes, we simply assume that all of the neutrinos are at $15 \mathrm{GeV}$ and are uniformly distributed throughout the pulse. Using these conditions for the NuMI beam, realistic conditions for the laser beam, and assuming a detection region about $1 \mathrm{~m}$ long, we estimate that there would be an event once every $10^{22} \mathrm{yr}$.

Given that our estimate of the time scale for detection of events is twelve orders of magnitude greater than the age of the universe, future work must turn towards astronomical and cosmological sources of neutrinos and fields. In astronomical phenomena where the magnetic field strengths can reach very high magnitudes, there exists the possibility of significant stimulation of this interaction. 


\section{Acknowledgments}

I am especially grateful to D. Dicus for his advice and suggestions throughout this work. I also wish to extend special thanks to T. Ditmire and G. Dyer for the useful information on ultrashort pulsed lasers, and to R. Zwaska for the beneficial conversations concerning the NuMI/MINOS experiment.

\section{APPENDIX: THE SCATTERING AMPLITUDE $\mathcal{M}_{\mathrm{n}}$}

As pointed out in the text, the scattering amplitudes $\mathcal{M}_{n}$ are related to the $S$-matrix by equation (18)

$$
S=\imath(2 \pi)^{4} \sum_{n=-\infty}^{\infty} \delta^{4}\left(q_{e}+q_{\bar{e}}+p_{\nu^{\prime}}-p_{\nu}-n k\right) \mathcal{M}_{n} .
$$

The scattering amplitudes are determined to be

$$
\begin{aligned}
\mathcal{M}_{n}= & -\frac{4 \pi \alpha}{2^{2} \cos ^{2} \theta_{\mathrm{W}} \sin ^{2} \theta_{\mathrm{W}}} \bar{u}_{s_{\nu^{\prime}}}\left(p_{\nu^{\prime}}\right) \gamma^{\sigma}\left(\frac{1}{2}-\frac{1}{2} \gamma^{5}\right) u_{s_{\nu}}\left(p_{\nu}\right) \frac{g_{\mu \sigma}-\left(p_{\nu}-p_{\nu^{\prime}}\right)_{\mu}\left(p_{\nu}-p_{\nu^{\prime}}\right)_{\sigma} /\left(m_{Z} c\right)^{2}}{\left(p_{\nu}-p_{\nu^{\prime}}\right)^{2}-\left(m_{Z} c\right)^{2}+\imath \Gamma_{Z} m_{Z} c^{2}} \\
& \times \bar{u}_{s_{e}}\left(p_{e}\right)\left[C_{0 n}(\zeta, \delta) \Gamma_{0}^{\mu}+C_{x n}(\zeta, \delta) \Gamma_{x}^{\mu}+C_{y_{n}}(\zeta, \delta) \Gamma_{y}^{\mu}+C_{x x n}(\zeta, \delta) \Gamma_{x x}^{\mu}+C_{x y_{n}}(\zeta, \delta) \Gamma_{x y}^{\mu}+C_{y y_{n}}(\zeta, \delta) \Gamma_{y y}^{\mu}\right] v_{s_{\bar{e}}}\left(p_{\bar{e}}\right)
\end{aligned}
$$

The $C_{n}(\zeta, \delta)$ 's are coefficients that depend on the Bessel functions

$$
\begin{aligned}
C_{0 n}(\zeta, \delta) & =J_{n}(\zeta) e^{\imath n \delta} \\
C_{x n}(\zeta, \delta) & =\frac{1}{2}\left(J_{n+1}(\zeta) e^{\imath(n+1) \delta}+J_{n-1}(\zeta) e^{\imath(n-1) \delta}\right) \\
C_{y_{n}}(\zeta, \delta) & =\frac{1}{2 \imath}\left(J_{n+1}(\zeta) e^{\imath(n+1) \delta}-J_{n-1}(\zeta) e^{\imath(n-1) \delta}\right) \\
C_{x x n}(\zeta, \delta) & =\frac{1}{2} J_{n}(\zeta) e^{\imath n \delta}+\frac{1}{4}\left(J_{n+2}(\zeta) e^{\imath(n+2) \delta}+J_{n-2}(\zeta) e^{\imath(n-2) \delta}\right) \\
C_{x y_{n}}(\zeta, \delta) & =\frac{1}{4 \imath}\left(J_{n+2}(\zeta) e^{\imath(n+2) \delta}-J_{n-2}(\zeta) e^{\imath(n-2) \delta}\right) \\
C_{y y_{n}}(\zeta, \delta) & =\frac{1}{2} J_{n}(\zeta) e^{\imath n \delta}-\frac{1}{4}\left(J_{n+2}(\zeta) e^{\imath(n+2) \delta}+J_{n-2}(\zeta) e^{\imath(n-2) \delta}\right)
\end{aligned}
$$

Recall that $\zeta$ and $\delta$ are defined through equations (13). The $\Gamma_{i}^{\mu}$ matrices are given by

$$
\begin{aligned}
\Gamma_{0}^{\mu} & =\gamma^{\mu}\left(-\frac{1}{2}+2 \sin ^{2} \theta_{\mathrm{W}}+\frac{1}{2} \gamma^{5}\right) \\
\Gamma_{x}^{\mu} & =-\frac{e a \gamma^{1} \not k}{2 p_{e} \cdot k} \Gamma_{0}^{\mu}-\Gamma_{0}^{\mu} \frac{e a \gamma^{1} \not k}{2 p_{\bar{e}} \cdot k} \\
\Gamma_{y}^{\mu} & =-\frac{e a \gamma^{2} \not k}{2 p_{e} \cdot k} \Gamma_{0}^{\mu}-\Gamma_{0}^{\mu} \frac{e a \gamma^{2} \not k}{2 p_{\bar{e}} \cdot k} \\
\Gamma_{x x}^{\mu} & =\frac{e a \gamma^{1} \not k}{2 p_{e} \cdot k} \Gamma_{0}^{\mu} \frac{e a \gamma^{1} \not k}{2 p_{\bar{e}} \cdot k} \\
\Gamma_{x x}^{\mu} & =\frac{e a \gamma^{1} \not k}{2 p_{e} \cdot k} \Gamma_{0}^{\mu} \frac{e a \gamma^{2} \not k}{2 p_{\bar{e}} \cdot k}+\frac{e a \gamma^{2} \not k}{2 p_{e} \cdot k} \Gamma_{0}^{\mu} \frac{e a \gamma^{1} \not k}{2 p_{\bar{e}} \cdot k} \\
\Gamma_{y y}^{\mu} & =\frac{e a \gamma^{2} \not k}{2 p_{e} \cdot k} \Gamma_{0}^{\mu} \frac{e a \gamma^{2} \not k}{2 p_{\bar{e}} \cdot k},
\end{aligned}
$$

where $\gamma^{1}$ and $\gamma^{2}$ are Dirac matrices.

[1] A. Erdas and G. Feldman, Nucl. Phys. B343, 597 (1990). 
[2] E. Elizalde, E. J. Ferrer, and V. de la Incera, Phys. Rev. D70, 043012 (2004), hep-ph/0404234.

[3] A. A. Gvozdev, N. V. Mikheev, and L. A. Vasilevskaya, Phys. Lett. B410, 211 (1997), hep-ph/9702285.

[4] D. A. Baiko and D. G. Yakovlev, Astron. Astrophys. 342, 192 (1999), astro-ph/9812071.

[5] D. Bandyopadhyay, S. Chakrabarty, and P. Dey, Phys. Rev. D58, 121301 (1998).

[6] L. B. Leinson and A. Perez, Phys. Lett. B518, 15 (2001), hep-ph/0110207.

[7] D. A. Dicus and W. W. Repko, Phys. Lett. B482, 141 (2000), hep-ph/0003305.

[8] R. Shaisultanov, Phys. Rev. Lett. 80, 1586 (1998), hep-ph/9709420.

[9] D. A. Dicus and W. W. Repko, Phys. Rev. Lett. 79, 569 (1997), hep-ph/9703210.

[10] D. A. Dicus, C. Kao, and W. W. Repko, Phys. Rev. D59, 013005 (1999), hep-ph/9806499.

[11] A. Abbasabadi, A. Devoto, D. A. Dicus, and W. W. Repko, Phys. Rev. D59, 013012 (1999), hep-ph/9808211.

[12] D. A. Dicus, K. Kovner, and W. W. Repko, Phys. Rev. D62, 053013 (2000), hep-ph/0003152.

[13] D. A. Dicus, W. W. Repko, and R. Vega, Phys. Rev. D62, 093027 (2000), hep-ph/0006264.

[14] S. J. Hardy and M. H. Thoma, Phys. Rev. D63, 025014 (2001), astro-ph/0008473.

[15] K. Bhattacharya and P. B. Pal (2002), hep-ph/0212118.

[16] A. A. Gvozdev, A. V. Kuznetsov, N. V. Mikheev, and L. A. Vassilevskaya, Phys. Atom. Nucl. 61, 1031 (1998), hep$\mathrm{ph} / 9710219$.

[17] G. G. Raffelt, Stars as Laboratories for Fundamental Physics: The astrophysics of neutrinos, axions, and other weakly interacting particles (The University of Chicago Press, Chicago, USA, 1996).

[18] C. Thompson and R. C. Duncan, Astrophys. J. 473, 322 (1996).

[19] T. Ditmire and G. Dyer, Private communication.

[20] M. M. Denisov and M. V. Fedorov, Sov. Phys. JETP 26, 779 (1968).

[21] C. Szymanowski, V. Véiard, R. Taieb, and A. Maquet, Physical Review A 56, 3846 (1997).

[22] D. M. Volkov, Z. Phys. 94, 250 (1935).

[23] D. M. Volkov, Zh. Eksp. Teor. Fiz. 7, 1286 (1937).

[24] V. B. Berestetskii, E. M. Lifshitz, and L. P. Pitaevskii, Quantum Electrodynamics, vol. 4 of Course of Theoretical Physics (Butterworth-Heinemann, Linacre House, Jordan Hill, Oxford OX2 8DP, 1982), 2nd ed.

[25] M. E. Peskin and D. V. Schroeder, An Introduction to Quantum Field Theory (Perseus Book Publishing, L.L.C., Cambridge, Massachusetts, 1995).

[26] E. W. Weisstein, Bessel function of the first kind, URL http://mathworld.wolfram.com/BesselFunctionoftheFirstKind.html

[27] J. J. Sakurai, Advanced Quantum Mechanics (Addison-Wesley, Reading, MA, 1967).

[28] S. E. Kopp, J. Phys. G29, 1757 (2003), hep-ex/0210009.

[29] R. Zwaska, Private communication. 